\title{
The Power of Low kV Microscopy in Sub-Micron Size Defect Analysis
}

\author{
Said Mansour and Cliff Alapa
}

JDS Uniphase, Science and Technology, 2789 Northpoint Parkway, Santa Rosa, CA 95407

Defects in optical coatings manifest themselves by scattering light from specimen surface. Their appearance usually depends on defect's point of origin, population and density. They normally appear to the eye as dark regions, as a haze or as a surface stain. In whatever appearance they have, their presence in display products in the age of digital mirrors and digital projection products is more than a nuisance. Their control and elimination are of primary concern to manufacturers as well as to product end users who spend big dollars in return of a high resolution and crisp clear display image. Accordingly, elimination of such defects is of prime challenge to coating companies such as ours. However, defect elimination starts by recognizing the defect's chemistry and the location of the defect with respect to the coating stack.

Defects in optical coatings have variety of flavors. They could originate at the substrate prior to coating as a result of substrate preparation. Typical defects at such stage are inherited substrate defects, scratches and polishing material residues. Substrate defects could also generate during substrate loading into coating machine causing tooling and/or coating flakes from previous runs to rain down on substrate. Furthermore, defects could generate during substrate soaking time prior to coating manifesting themselves as a physical and/or chemical surface changes. In all previous cases, defects get buried under the coating stack and consequently reside at the bottom of coating stack. Defects could also be generated during film vapor deposition where a seed particle is ejected as a result of coating irregularity by target material or power supply [1]. Another source of optical coating defects, could occur after coating is complete during vacuum chamber venting, surface handling, packaging or shipping. A combination of one or more type of defects is also possible. The size of defects normally varies. Their range could be from a few microns down to submicron sizes. Large defects are easy to recognize and easy to control. However, recognition and identification of smaller defects are a bit of a challenge.

Scanning electron microscopy and microanalysis is method of choice for quick and easy identification of coating defects. However, electron beam conditions become critical when analyzing small defects. High accelerating e-beam voltage provides high source brightness and ease in adjusting image that made the use of high accelerating voltages a popular practice amongst SEM users. Furthermore, high energies provide longer electron beam range useful in analysis of buried defects. However, high e-beam voltage has many shortcomings, amongst which an increase in charging and large interaction volume that could result in bulk rather than localized analysis. It is thus logical to suggest low e-beam energies be used in surface sub-micron size defect analysis. The electron range falls from microns to a few nanometer as penetration depth falls as $\mathrm{E}^{-3.5}(\mathrm{E}-$ is electron beam energy), spatial resolution improves, and near-surface information dominates [2].

EDS elemental microanalysis of submicron defects is normally carried out by comparing defect spectrum with a defect-free (normal) area. Figures 1 and 2, show SEM images of 2- submicron surface defect in a multilayer coating. The upper most layer is $\mathrm{MgF} 2$ layer, while layers underneath are oxides of Ti, Pr, and Al. The corresponding EDS spectra collected at $10 \mathrm{keV}$ and $5 \mathrm{keV}$ e-beam 
energies are also shown. Upon comparison of defect spectrum with a normal area, very little difference was found between the 2- spectra at $10 \mathrm{kV}$ e-beam energies. This leaves the analyst with more than one possible conclusion, probably false ones. One possible conclusion, defect is buried at a depth that exceeds e-beam energy range. Another possible conclusion is defect is a coating flake and thus having composition similar to coating materials. A third possible conclusion, defect is an extremely thin MgF2 flake. Figure 2, on the other hand, shows EDS spectrum of a similar defect using $5 \mathrm{kV}$ beam energy (a neighboring defect was used in order to minimize carbon deposition). Contrary to $10 \mathrm{kV}$ spectrum where $\mathrm{Mg}$ and $\mathrm{F}$ lines in addition to $\mathrm{Pr}, \mathrm{Al}$, and $\mathrm{O}$ elemental lines corresponding to subsurface coating layers, $5 \mathrm{kV}$ spectrum shows $\mathrm{Mg}$ and $\mathrm{F}$ lines only (C-peak is due to surface contamination while Au was used as a conductive film). Furthermore, spectrum in figure 2 shows a decrease in $\mathrm{Mg}$ line intensity and an increase in $\mathrm{F}$ - and $\mathrm{C}$ - signals. Contrary to a mistaken earlier interpretation using high e- beam energy, the defect turns out to be a florinated hydrocarbon material having F- as one of its constituents. The spectrum further suggests that the defect is a surface defect resulting in suppression of $\mathrm{Mg}$ and $\mathrm{F}$ signals from $\mathrm{MgF} 2$ top layer. The paper will further discuss other types of sub-micron size defects and the role of low e-beam energies in their recognition and sources.
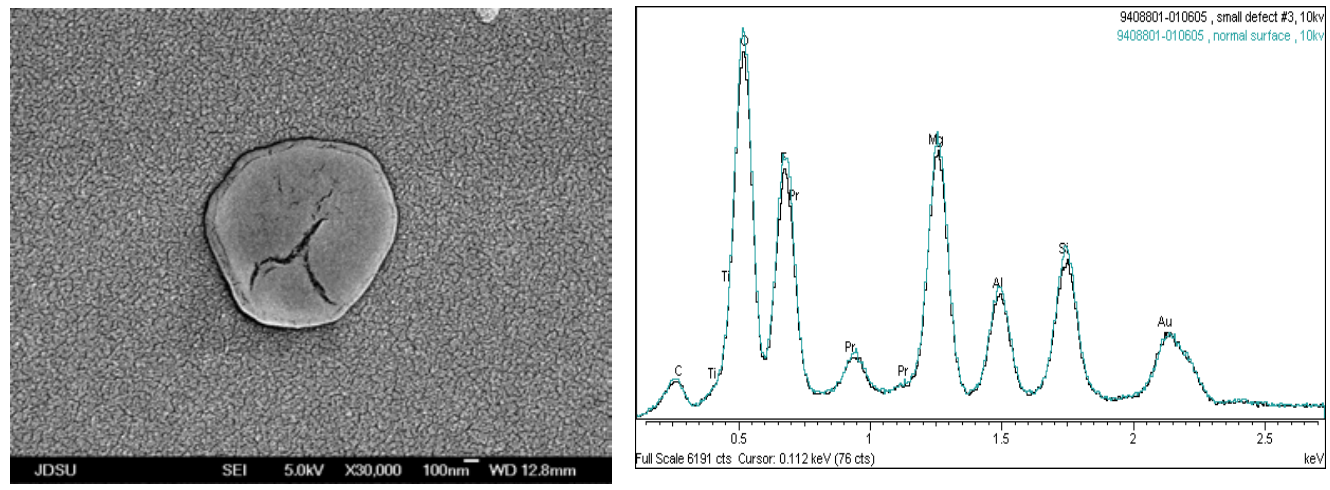

Figure 1: SEM image and corresponding EDS spectrum taken using $10 \mathrm{kV}$ e- beam energy of a submicron surface defect on top of a multilayer coated surface.
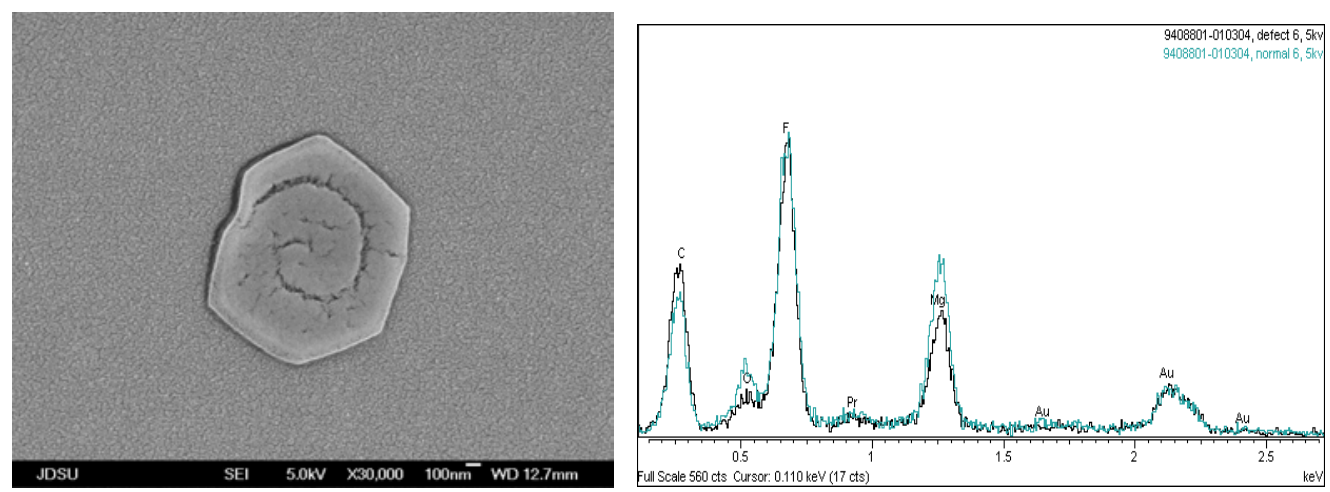

Figure 2: SEM image and corresponding EDS spectrum taken using $5 \mathrm{kV}$ e- beam energy of a neighboring sub-micron surface defect next to defect shown in figure 1.

\section{References}

[1] R.J Tench,R. Chow, and M.R. Kozlowski, J. Vac. Sci. Technol. A 12 (1994) 2808

[2] D. Joy and D. Newbury, Microsc. Microanal. 7 (2001) 762 